\title{
An Electronic Hybrid with Adaptive Balancing for Telephony
}

\author{
DAVID G. MESSERSCHMITT, SENIOR MEMBER, IEEE
}

\begin{abstract}
A new form of adaptive balancing hybrid is proposed for application to the two-wire to four-wire interface of a local digital switch. It is similar to an echo canceller but with a single degree of freedom and much simpler circuitry. In this paper a theory of operation of the hybrid is developed, and in a companion paper two circuit implementations and experimental results are reported.
\end{abstract}

\section{INTRODUCTION}

W ITH the advent of digital switches for replacement of metallic crosspoint analog switches in the local telephone network, there is a need for more accurate balancing of the hybrid used for two-wire to four-wire conversion. The function of the hybrid, illustrated in Fig. 1, is to convert from the inherently four-wire transmission in the local digital switch to the balanced two-wire transmission of the subscriber loop. Specifically, it ideally provides a large loss (called the singing margin) around the closed path consisting of both hybrids and the four-wire transmission path, thereby preventing singing and minimizing echo effects. It would be desirable to have nominally $0 \mathrm{~dB}$ insertion loss between the two-wire ports (the same as for two-wire analog space-division switches). The need for more accurate balancing arises because previous applications of four-wire transmission to the toll network were always associated with nonzero insertion loss, with a corresponding increase in the singing margin.

There have been many solutions proposed to this problem. One is to allow a switch insertion loss (usually about $2 \mathrm{~dB}$ ), but this adversely affects loss-noise grade of service on the longer subscriber loops [2]. Another approach is to replace the former single compromise hybrid termination with two or more terminations tailored to each type of subscriber loop design (the accuracy with which the termination matches the actual loop impedance governs the hybrid balance). Two independent studies [1]-[4] have concluded that two such terminations, one for loaded loops and another for nonloaded loops, will result in acceptable singing margin with a zero-loss switch. Approaches to choosing these terminations have included manual switch setting from plant records, automatic setting by transmitting a tone and measuring the return loss during call setup, and automatic on-hook impedance measurement.

In this paper, we propose an automatic hybrid balancing

Paper approved by the Editor-in-Chief of the IEEE Communications Society for publication after presentation at the International Conference on Communications, Seattle, WA, June 1980. Manuscript received October 5, 1979; revised March 12, 1980. This work was supported by a grant from TRW VIDAR, Mountain View, CA.

The author is with the Department of Electrical Engineering and Computer Sciences and the Electronics Research Laboratory, University of California, Berkeley, CA 94720.

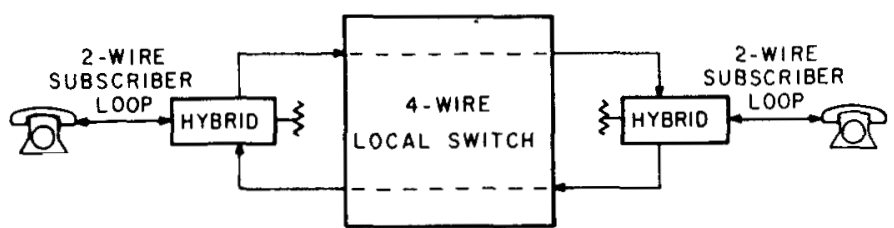

Fig. 1. Function of hybrid at local switch subscriber loop interface.

approach which is similar in philosophy to an adaptive echo canceller of primary interest in satellite transmission [5] in that it continually adapts on the basis of virtually any input signal, including speech. It is, however, considerably simpler than the echo canceller, and hence would consume considerably less die area and power in an integrated circuit implementation (a factor of some consequence since the circuit may be replicated on a per-subscriber loop basis). In addition, its severely constrained degrees of freedom ensure that a fair degree of balance can be guaranteed regardless of the state of adaptation. In contrast, an echo canceller is capable of much better balance after convergence, but when misadjusted could also readily provide a very poor balance, resulting in a singing state from which recovery is problematic. Of course, an echo canceller tap-weight vector could presumably also be suitably constrained to obviate singing, although the constraint boundaries are likely to be somewhat complex.

In Section II we will describe the adaptive balancing hybrid, and in Section III we will analyze various aspects of the behavior of the hybrid, including speed of adaptation, asymptotic transhybrid loss, effect of limiting on the adaptation, the interaction of back-to-back hybrids, the effect of the far-end talker spectrum, and performance of a particular near-end talker detector. A much more detailed analysis of the convergence properties of the echo canceller, which is applicable with minor modification to the adaptive balancing hybrid, is summarized in [12].

\section{DESCRIPTION OF THE ADAPTIVE BALANCING HYBRID}

There are many ways to implement the hybrid function, but we concentrate on the particular configuration of Fig. 2(a). The subscriber loop with characteristic impedance $Z_{L}$ is assumed to be coupled to the switch through a transformer to convert from balanced to unbalanced (it should be emphasized that other transformerless realizations would not preclude our approach to adaptive balancing). The transformer winding on the switch side has superimposed on it both the near-end talker $y(t)$, as well as a filtered version of $x(t)$, the far-end talker. The cancelling filter $H(\omega)$ is designed to emulate the transfer function from $x(t)$ to the transformer, and thus cancel the com- 


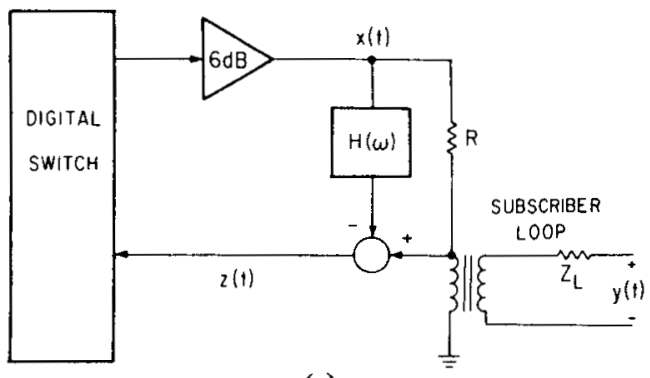

(a)

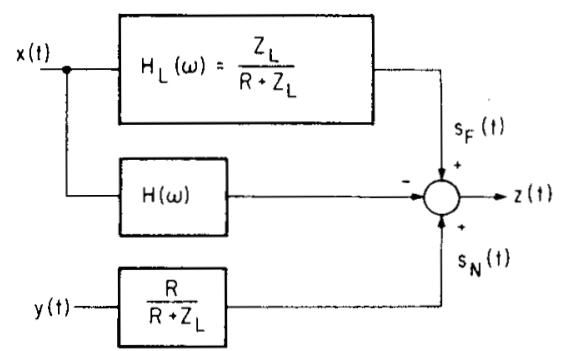

(b)

Fig. 2. Electronic hybrid. (a) Electronic hybrid circuit; (b) equivalent circuit.

ponent of $x(t)$ which would otherwise appear in $z(t)$. Finally, the $6 \mathrm{~dB}$ gain compensates for the loss between $x(t)$ and the transformer. This loss will be nominally $6 \mathrm{~dB}$ since $R$ is the impedance presented to the loop by the hybrid, and thus will be chosen to be $900 \Omega$, the nominal loop impedance.

An equivalent circuit for the hybrid is shown in Fig. 2(b), where the signal $y(t)$ is the near-end talker originating on the subscriber loop. An infinite transhybrid loss is achieved if $H(\omega)$ is chosen to be $H_{L}(\omega)$, but $Z_{L}$ is unfortunately not known precisely. Nevertheless, previous work [1]-[4] has indicated that adequate singing margin is achieved if $H(\omega)$ is chosen to be $H_{1}(\omega)$ or $H_{2}(\omega)$, where

$$
H_{j}(\omega)=\frac{Z_{j}}{R+Z_{j}}, \quad j=1,2
$$

and $Z_{1}$ and $Z_{2}$ are two impedances chosen to match loaded and nonloaded loops. Our approach is then to let

$$
H(\omega)=\theta H_{1}(\omega)+(1-\theta) H_{2}(\omega)
$$

where $\theta$ is chosen in the range $0 \leqslant \theta \leqslant 1$. When $\theta=1, H(\omega)=$ $H_{1}(\omega)$, and when $\theta=0, H(\omega)=H_{2}(\omega)$; thus, judicious choice of $\theta$ should yield adequate singing margin (in fact, availability of values of $\theta$ between 0 and 1 should yield an even better singing margin distribution than determined in [1]-[4].) The resulting transhybrid transfer function [from $x(t)$ to $z(t))$ ] is easily shown to be

$$
\begin{aligned}
H_{\mathrm{TH}}(\omega) & =H_{L}(\omega)-\theta H_{1}(\omega)-(1-\theta) H_{2}(\omega) \\
& =R \frac{\left(R+Z_{1}\right)\left(Z_{L}-Z_{2}\right)+\theta\left(R+Z_{L}\right)\left(Z_{2}-Z_{1}\right)}{\left(R+Z_{L}\right)\left(R+Z_{1}\right)\left(R+Z_{2}\right)} .
\end{aligned}
$$

Specific values for $Z_{1}$ and $Z_{2}$ are recommended in [1]-[4] .
The question remains as to how $\theta$ can be adjusted automatically. Assuming $x(t)$ and $y(t)$ are wide-sense stationary random processes (at least in the short term), we define the power in $z(t)$, which is a function of $\theta$, to be $P(\theta)$ :

$$
P(\theta)=E\left[z^{2}(t)\right] \text {. }
$$

Our criterion for choice of $\theta$ will be to minimize $P(\theta)$, or in other words, to minimize the power of the far-end talker appearing in $z(t)$. It should be recognized that the resulting $\theta$ will depend on the spectral characteristics of $x(t)$; however, as will be demonstrated later, this dependence is not marked because of the severely limited degrees of freedom of the adapting cancelling filter.

How do we build a circuit which determines the $\theta$ which minimizes (4)? First we recognize that $P(\theta)$ will be a quadratic function of $\theta$ :

$$
P(\theta)=P\left(\theta_{0}\right)+K\left(\theta-\theta_{0}\right)^{2} .
$$

This dependence, together with explicit expressions for $P\left(\theta_{0}\right)$, $\theta_{0}$, and $K$, is derived in Appendix $\mathrm{A}$. The unique minimizing $\theta$ is $\theta=\theta_{0}$. Defining $Q(\theta)$ to be

$$
Q(\theta)=-\frac{1}{2} \frac{d P(\theta)}{d \theta}=K\left(\theta_{0}-\theta\right)
$$

we recognize that if a circuit could be constructed to estimate $Q(\theta)$, the appropriate adjustment of $\theta$ would be to increase $\theta$ if $Q(\theta)>0$ and decrease $\theta$ if $Q(\theta)<0$. Substituting (4) into (6)

$$
\begin{aligned}
Q(\theta) & =-\frac{1}{2} \frac{d}{d \theta} E\left(z^{2}(t)\right) \\
& =-E\left(z(t) \frac{d z(t)}{d \theta}\right) \\
& =E(z(t) w(t))
\end{aligned}
$$

where $w(t)$ is the output of a filter with transfer function $\left(H_{1}(\omega)-H_{2}(\omega)\right)$ and input $x(t)$.

The resulting adaptive cancelling filter is shown in Fig. 3. First $w(t)$ is formed, and then an estimate of $Q(\theta)$ is formed from (7) by multiplying $w(t)$ and $z(t)$. The result is

$$
w(t) z(t)=q(t)+Q(\theta)
$$

where $q(t)$ is a zero-mean "noise" in the estimate of $Q(\theta)$. Since from (6) $Q(\theta)$ is proportional to the difference between the optimum and actual $\theta$, the natural processing is to integrate this $Q(\theta)$ estimate to determine $\theta$. If there is an initial error in $\theta$, the integrator will eventually reduce that difference to zero. Finally, there is a switch which disconnects the integrator and thereby disables adaptation and retains the last value of $\theta$ at the integrator output when a speech detector indicates that there is a near-end speaker, or no far-end speaker, or both. The need for a far-end speaker for adaptation to proceed is evident, but the need to disable adaptation while there 


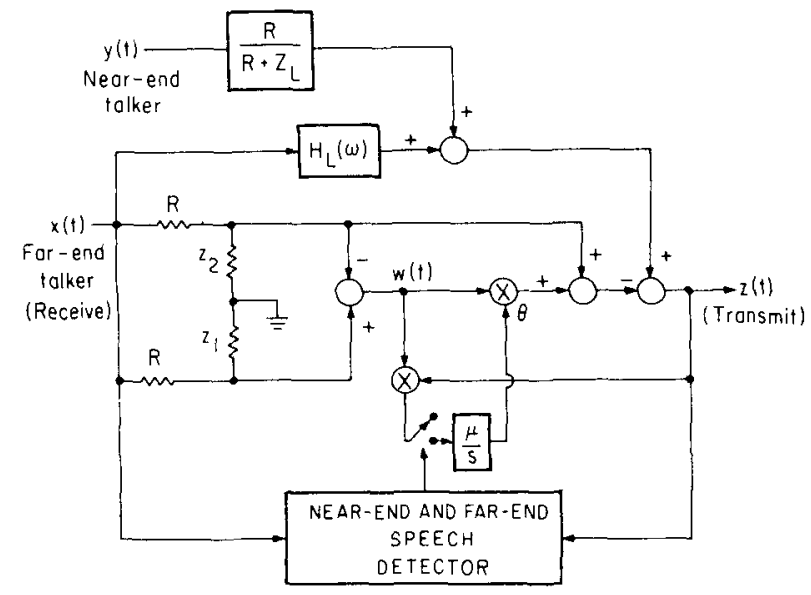

Fig. 3. Adaptive balancing hybrid.

is a near-end speaker is less obvious and is due to two difficulties:

1) A near-end speaker will directly cause a large variance (but not bias) in the estimate of $Q(\theta)$, since it appears in $z(t)$, and

2) A delayed and filtered version of the near-end speaker will appear in $x(t)$ due to the presumably finite far-end hybrid return loss, causing a bias in the estimate of $Q(\theta)$.

Both these issues will be examined in further detail in the sequel.

\section{ANALYSIS OF THE HYBRID}

This section will develop relations useful in understanding the behavior of the adaptive balancing hybrid.

\section{A. Single Hybrid Analysis}

In Appendix A, the parameters of ( 5 ) are determined explicitly, including $P\left(\theta_{0}\right), \theta_{0}$, and $K$. The most important conclusion is that

$$
K=\sigma_{w}{ }^{2},
$$

the variance of $w(t)$. Thus, the size of $Q(\theta)$ is directly proportional to $\sigma_{w}{ }^{2}$, and in order to interpret $Q(\theta)$ in terms of the size of adjustment to $\theta$ which must be made to achieve $\theta_{0}$, $\sigma_{w}{ }^{2}$ should be separately estimated.

\section{B. Speed of Adaptation}

An equivalent circuit to the hybrid adaptation is shown in Fig. 4. The input to the integrator reflects (6) and (8), and the output is $\theta(t)$. The response of the circuit to an initial condition $\theta(0)$ is readily found to be (with $q(t)=0$ )

$$
\theta(t)=\theta_{\mathrm{G}}\left(1-e^{-K \mu t}\right)+\theta(0) e^{-K \mu t} .
$$

Thus the speed of exponential adaptation can be increased by increasing the integrator gain $\mu$. In view of (9), the speed of adaptation also increases as the power of the far-end talker $x(t)$ increases.

\section{Variance of $\theta$}

The $\theta$ estimate of Fig. 4 also reflects the noise $q(t)$. The

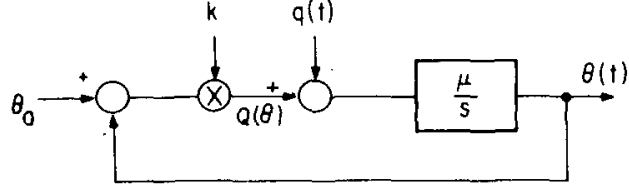

Fig. 4. Model of hybrid adaptation.

transfer function between $q(t)$ and $\theta(t)$ is

$$
G(s)=\frac{\mu}{s+K_{\mu}}
$$

and therefore the variance of the $\theta$ estimate is

$$
\operatorname{var}(\theta)=\int_{-\infty}^{\infty} \frac{\mu^{2} S_{q}(\omega)}{\omega^{2}+K^{2} \mu^{2}} \frac{d \omega}{2 \pi}
$$

Under most circumstances the cutoff frequency $K \mu$ will be quite small relative to the bandwidth of $q(t)$, and thus $S_{q}(\omega)$ can be approximated by $S_{q}(0)$,

$$
\operatorname{var}(\theta) \cong \frac{\mu}{2 K} S_{q}(0)
$$

Notice that speeding up adaptation by increasing $\mu$ also increases the estimate variance. The dependence of (13) on the power in $x(t)$ is more complicated, since both $S_{q}(0)$ and $K$ are dependent on that power.

Relations for $S_{q}(0)$ are obtained in Appendix B for several cases. When $x(t)$ is sinusoidal

$$
x(t)=A \cos \left(\omega_{0} t+\phi\right)
$$

and $\phi$ is uniformly distributed between $-\pi$ and $\pi$, then

$$
S_{q}(0)=\frac{1}{2} A^{2}\left|H_{1}\left(\omega_{0}\right)-H_{2}\left(\omega_{0}\right)\right|^{2} S_{y}\left(\omega_{0}\right) .
$$

When $y(t)$ is sinusoidal as in (14)

$$
S_{q}(0)=\frac{1}{2} A^{2}\left|H_{1}\left(\omega_{0}\right)-H_{2}\left(\omega_{0}\right)\right|^{2} S_{x}\left(\omega_{0}\right)
$$

Finally, when $x(t)$ and $y(t)$ are independent stationary Gaussian random processes, which is probably the closest tractable model for speech signals, then

$$
S_{q}(0)=\sigma_{N}^{2}+\sigma_{F}^{2}
$$

where $\sigma_{N}^{2}$ is the variance due to the near-end talker,

$$
\sigma_{N}^{2}=\int_{-\infty}^{\infty} S_{x}(\omega) S_{y}(\omega)\left|H_{1}(\omega)-H_{2}(\omega)\right|^{2} \frac{d \omega}{2 \pi}
$$

and $\sigma_{F}{ }^{2}$ is the variance due to the far-end talker alone,

$$
\sigma_{F}^{2}=2 \int_{-\infty}^{\infty} S_{x}^{2}(\omega)\left[\operatorname{Re} H_{T}(\omega)\right]^{2} \frac{d \omega}{2 \pi}
$$


where

$$
\begin{aligned}
H_{T}(\omega)= & {\left[H_{L}(\omega)-H_{2}(\omega)\right] *\left[H_{1}(\omega)-H_{2}(\omega)\right] } \\
& -\theta\left|H_{1}(\omega)-H_{2}(\omega)\right|^{2} .
\end{aligned}
$$

If the power spectrum of $x(t)$ retains its same shape but the total power varies, then all the terms in (15)-(17) increase in direct proportion to that power except for (19), which varies as the square of that power. Similarly, $K$ varies in proportion to the power in $x(t)$ from (9). Thus, the variance of (13) tends to be independent of the power in $x(t)$ except for (19), which will cause a term in the variance to increase in direct proportion to the power in $x(t)$.

Another conclusion from (14)-(19) is that when either $x(t)$ or $y(t)$ is sinusoidal, significant variation in $\theta$ occurs only when the other signal has significant components at the same frequency. When $x(t)$ is Gaussian, there is some variation in $\theta$ even when $y(t)$ is zero. When $y(t)$ is then also Gaussian, there is an additional variation due to the interaction of $x(t)$ and $y(t)$ which is proportional to the integral of $S_{x}(\omega) S_{y}(\omega)$, and thus is significant only when $x(t)$ and $y(t)$ have significant frequency components in common.

\section{Effect of Limiting in Crosscorrelation}

A considerably simpler circuit implementation results if, instead of averaging $w(t) z(t)$, we hard-limit either $w(t)$ or $z(t)$ or both. Again, the effect of this modification can be evaluated by assuming that $w(t)$ and $z(t)$ are Gaussian. The results of this analysis are rather detailed and relegated to Appendix C.

The general conclusion of Appendix $\mathrm{C}$ is that limiting $z(t)$ is undesirable, since the sensitivity to a near-end talker is greatly accentuated (the speed of adaptation is reduced and the variance of $\theta$ is increased). On the other hand, limiting $w(t)$ has the more modest impact of making the variance of $\theta$ more sensitive and the speed of adaptation less sensitive to far-end talker power. It is therefore recommended that $w(t)$ be limited.

\section{E. Effect of Far-End Talker Spectrum}

As previously mentioned, the optimum value of $\theta, \theta_{0}$, is dependent on $S_{x}(\omega)$. We can understand the degree of dependence by taking $x(t)$ to be a sinusoid with radian frequency $\omega_{0}$, in which case $\theta_{0}$ is given by (A.6) as

$$
\theta_{0}=\left.\frac{\operatorname{Re}\left(H_{L}-H_{2}\right)\left(H_{1}-H_{2}\right)}{\operatorname{Re}\left(H_{1}-H_{2}\right)^{2}}\right|_{\omega=\omega_{0}}
$$

independent of the amplitude of the sinusoid. It has been recommended [1] , [2] that $Z_{1}$ and $Z_{2}$ be parallel $R C$ networks,

$$
H_{j}\left(\omega_{0}\right)=\frac{1}{j \omega_{0} R C_{j}+\frac{R}{R_{j}}+1}, \quad j=1,2,
$$

where $R=900, R_{1}=1650, R_{2}=800, C_{1}=5 \mathrm{nF}$, and $C_{2}=$ $50 \mathrm{nF}$. To study the effect of $\omega_{0}$ on (21) we take $Z_{L}$ to be the average over a random sample of customer loops given in [7] and tabulated in Table I.

\begin{tabular}{|c|c|c|}
\hline \multirow[t]{2}{*}{ Erequency $(\mathrm{kHz})$} & \multicolumn{2}{|c|}{ Impedance (Ohms) } \\
\hline & Non-loaded & Loaded \\
\hline 0.2 & $800+j 200$ & $1550+j 210$ \\
\hline 1.0 & $900-j 330$ & $1050-j 280$ \\
\hline 3.2 & $410-j 370$ & $2200+j 50$ \\
\hline
\end{tabular}

TABLE I

AVERAGE SUBSCRIBER-LOOP IMPEDANCE VERSUS FREQUENCY

The resulting $\theta_{0}$ is tabulated in Table II. Note that when $\theta_{0}<0$ or $\theta_{0}>1$, the circuitry will choose $\theta=0$ or $\theta=1$, respectively. At the band edges, 200 and $3200 \mathrm{~Hz}$, the value of $\theta_{0}$ is very close to the expected $\theta=0$ for nonloaded loops and $\theta=1$ for loaded loops. At the band center, $1000 \mathrm{~Hz}, \theta_{0}$ is near the center of the range for both cases. Thus, we would expect a significant variation in $\theta_{0}$ depending on the type of signal. For example, speech will have a spectrum concentrated near the bottom of the band, while voiceband data will be concentrated near the center. We would not however expect a large variation in $\theta_{0}$ for a single type of signal, particularly if the convergence time of the adaptation is long.

This dependence of $\theta_{0}$ on frequency is a result of the adaptive hybrid's attempt to obtain the best balance (which is a function of $S_{x}(\omega)$ as well as the loop impedance). In fact, this feature enables the adaptive hybrid to obtain a better balance than could be obtained by simple loop segregation alone. Finally, the echo canceller does not have this sensitivity to $S_{x}(\omega)$ because its degrees of freedom are adequate to obtain an essentially "perfect" balance over all frequencies (subject to limitation of quantization noise, etc.).

\section{F. Back-to-Back Hybrids}

When two adaptive balancing hybrids are placed back-toback with an intervening four-wire path, there will be a mutual interaction which we would expect to become more severe as the singing margin decreases.

A model for the situation is shown in Fig. 5. $H_{\mathrm{TH}, 1}(\omega)$ is the transhybrid loss of the near-end hybrid, and $H_{\mathrm{TH}, 2}(\omega)$ the far-end hybrid. The near-end talker is $y_{1}(t)$ and the far-end talker $y_{2}(t)$. Finally, the four-wire path is modeled by transfer function $H_{s}(\omega)$, the most important components of which are: 1) a $6 \mathrm{~dB}$ gain to compensate for the hybrid four-wire to twowire loss (when $0 \mathrm{~dB}$ insertion loss is desired); 2) low-and highpass filtering due to the PCM antialiasing and reconstruction filters; and 3) delay of 1-ms or more due to the filters and the buffers, time-slot interchange, etc., of the digital switch.

If we define the singing margin transfer function (we suppress the $\omega$ dependence)

$$
H_{\mathrm{SM}}(\omega)=H_{S}{ }^{2} H_{\mathrm{TH}, 1} H_{\mathrm{TH}, 2}
$$

then the transfer functions from the two talkers to $w_{1}(t)$ and $z_{1}(t)$ are

$$
\begin{aligned}
& \frac{Z_{1}(\omega)}{Y_{1}(\omega)}=\frac{1}{1-H_{\mathrm{SM}}} \\
& \frac{W_{1}(\omega)}{Y_{1}(\omega)}=\frac{H_{S}^{2} H_{\mathrm{TH}, 2}\left(H_{1}-H_{2}\right)}{1-H_{\mathrm{SM}}}
\end{aligned}
$$


TABLE II

OPTIMUM $\theta$ FOR IMPEDANCES OF TABLE I

\begin{tabular}{ccc} 
Frequency (kHz) & \multicolumn{1}{c}{$\theta_{0}$} \\
& $\frac{\text { Non-1oaded }}{2}$ & Loaded \\
0.2 & 0.0188 & 0.9226 \\
1.0 & 0.3576 & 0.5045 \\
3.2 & -0.1664 & 1.2400 \\
\hline
\end{tabular}

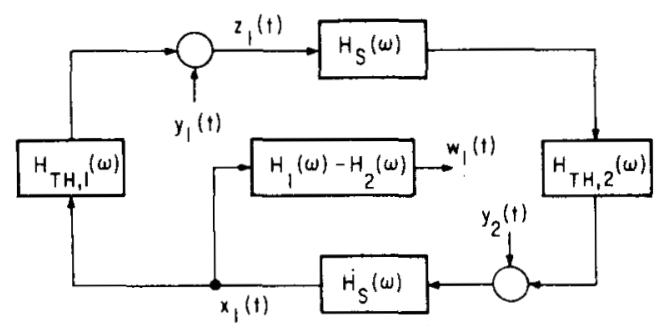

Fig. 5. Model of back-to-back hybrid interaction.

$$
\begin{aligned}
& \frac{Z_{1}(\omega)}{Y_{2}(\omega)}=\frac{H_{S} H_{\mathrm{TH}, 1}}{1-H_{\mathrm{SM}}} \\
& \frac{W_{1}(\omega)}{Y_{2}(\omega)}=\frac{H_{S}\left(H_{1}-H_{2}\right)}{1-H_{\mathrm{SM}}} .
\end{aligned}
$$

It follows that the cross-power spectral density between $z_{1}(t)$ and $w_{1}(t)$ is

$$
\begin{aligned}
S_{z_{1} w_{1}}(\omega)= & S_{y_{1}}(\omega) \frac{H_{S}{ }^{2} H_{\mathrm{TH}, 2}\left(H_{1}-H_{2}\right)}{\left|1-H_{\mathrm{SM}}\right|^{2}} \\
& +S_{y_{2}}(\omega) \frac{\left|H_{S}\right|^{2} H_{\mathrm{TH}, 1} *\left(H_{1}-H_{2}\right)}{\left|1-H_{\mathrm{SM}}\right|^{2}} .
\end{aligned}
$$

This density can be used to predict the behavior of the hybrids under various conditions.

The second term of (27) is the desired one which enables adaptation of $\theta$. When we recognize $S_{y_{2}}\left|H_{S}\right|^{2}$ as the far-end talker spectrum analogous to $S_{x}$, then the only modification due to the interaction of hybrids is the denominator term. Since we are concerned with the effect on the $\theta$ of the converged hybrid, assume that the hybrids are able to achieve an $8 \mathrm{~dB}$ singing margin, so that $\left|H_{\mathrm{SM}}\right| \leqslant 0.398$. It is then simple to show that

$$
0.512 \leqslant \frac{1}{\left|1-H_{\mathrm{SM}}\right|^{2}} \leqslant 2.76
$$

Thus the effect of the denominator term in (27) is to accentuate or attenuate those frequencies where the singing margin is poor by at most 4.4 or $2.9 \mathrm{~dB}$, respectively.

The first term in (27) will result in an undesired bias in $\theta$ due to a finite transhybrid loss for the far-end hybrid. We can get some idea of the relative size of the two terms by taking the ratio of the first to the second:

$$
\frac{S_{y_{1}}(\omega)}{S_{y_{2}}(\omega)} \frac{H_{S}^{2}}{\left|H_{S}\right|^{2}} \frac{H_{\mathrm{TH}, 2}}{H_{\mathrm{TH}, 1}} \text {. }
$$

The second term has magnitude unity, while the third could easily have magnitude greater than unity. Thus, to keep the ratio small, we must ensure that the first term is small, that is, we must keep the near-end talker power small relative to the far-end talker power. The only way to do this is to disable adaptation when the near-end talker is comparable to or larger than the far-end talker, necessitating the speech detector shown in Fig. 3.

\section{G. Power-Estimation Speech Detector}

A standard method of speech detection would be to observe the voltages $x(t)$ and $z(t)$, declaring near-end talker when $z(t)$ becomes appreciable to or larger than $x(t)$ in some sense [5]. This method is somewhat sensitive to the degree of convergence of the hybrid adaptation, since that will influence the component of $x(t)$ which appears in $z(t)$. In contrast to the toll network [5], the minimum singing margin prior to adaptation will be much smaller than $6 \mathrm{~dB}$.

A. de la Plaza [8] has suggested an alternative method of speech detection which avoids this convergence sensitivity completely. The method can be understood in terms of the equivalent circuit of the hybrid of Fig. 6(a). The near-end talker $y(t)$ can be distinguished from the far-end talker $x(t)$ due to the fact that the power due to the former is flowing out of the load impedance $Z_{L}$, whereas the power due to the latter is flowing into the load impedance. The instantaneous power flow is $i(t) u(t)$. The circuit of Fig. $6(\mathrm{~b})$ estimates the direction of short term power by cross correlating $v(t)$ (which is proportional to $i(t))$ with $u(t)$. When the cross correlation is zero, it indicates approximately equal near-end and far-end talker powers; when it is positive it indicates a larger far-end talker, and vice versa when it is negative. Since we want to distinguish small near-end talker powers, the threshold applied to the cross correlation will generally be positive, with adaptation of the hybrid proceeding when that threshold is exceeded.

A related technique was independently proposed in [9] in the context of a study of talker powers in the telephone network.

Assuming $x(t)$ and $y(t)$ are uncorrelated, it is readily shown from the equivalent circuit of Fig. $6(\mathrm{c})$ that the relevant power spectra are

$$
\begin{aligned}
& S_{u v}(\omega)=\frac{R}{\left|R+Z_{L}\right|^{2}}\left(Z_{L} S_{x}(\omega)-R S_{y}(\omega)\right), \\
& S_{u}(\omega)=\frac{1}{\left|R+Z_{L}\right|^{2}}\left(\left|Z_{L}\right|^{2} S_{x}(\omega)+R^{2} S_{y}(\omega)\right), \\
& S_{v}(\omega)=\frac{R^{2}}{\left|R+Z_{L}\right|^{2}}\left(S_{x}(\omega)+S_{y}(\omega)\right) .
\end{aligned}
$$

If it is assumed that $Z_{L}$ is resistive, the cross-correlation $R_{u v}(0)$ is readily determined in terms of $\sigma_{x}{ }^{2}$ and $\sigma_{y}{ }^{2}$. However, for the more realistic case where $Z_{L}$ is complex and frequency dependent, $R_{u v}(0)$ is dependent on the shape of $S_{x}(\omega)$ and $S_{y}(\omega)$. To simplify matters, we can assume that $x(t)$ and $y(t)$ are both narrow-band processes centered at frequency $\omega_{0}$. For this case, where

$$
Z_{L}\left(\omega_{0}\right)=R_{L}+j X_{L}
$$




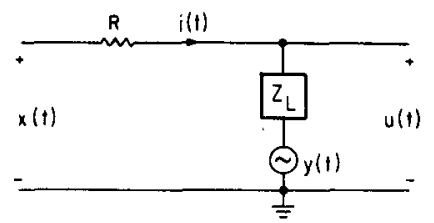

(a)

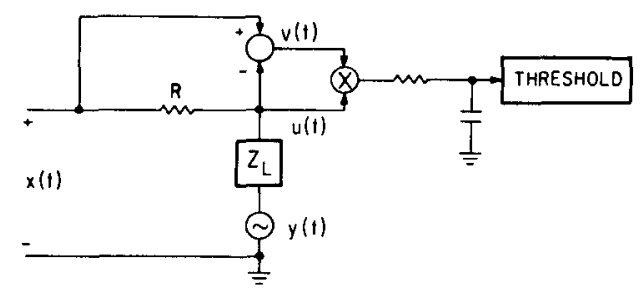

(b)

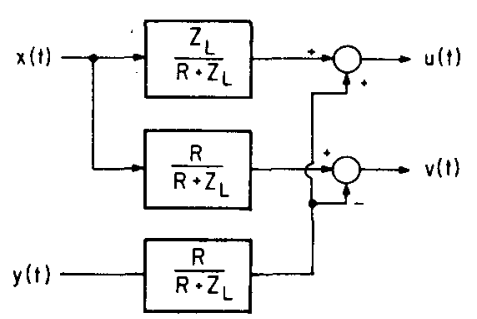

(c)

Fig. 6. Power-estimation speech detector.

we obtain from (28)-(30)

$$
\begin{aligned}
& R_{u v}(0)=\frac{R}{\left(R+R_{L}\right)^{2}+X_{L}^{2}}\left(R_{L} \sigma_{x}^{2}-R{\sigma_{y}}^{2}\right) \\
& R_{u}(0)=\frac{1}{\left(R+R_{L}\right)^{2}+X_{L}{ }^{2}}\left(\left(R_{L}^{2}+X_{L}^{2}\right) \sigma_{x}^{2}+R^{2}{\sigma_{y}}^{2}\right)
\end{aligned}
$$

$$
R_{v}(0)=\frac{R^{2}}{\left(R+R_{L}\right)^{2}+X_{L}^{2}}\left(\sigma_{x}^{2}+\sigma_{y}{ }^{2}\right) .
$$

For reasons which will soon be apparent, as pointed out by de la Plaza [8] it is advantageous to limit both $u(t)$ and $v(t)$ prior to cross correlation, so that we estimate

$$
\rho \triangleq E(\operatorname{sgn} u(t) \operatorname{sgn} v(t))
$$

If we assume that $u(t)$ and $v(t)$ are Gaussian, then as in (C.3)

$$
\begin{aligned}
\rho & =\frac{2}{\pi} \sin ^{-1}\left(\frac{R_{u v}(0)}{\sqrt{R_{u}(0) R_{v}(0)}}\right) \\
& =\frac{2}{\pi} \sin ^{-1} \frac{R_{L} \sigma_{x}{ }^{2}-R \sigma_{y}{ }^{2}}{\sqrt{\left[\left(R_{L}^{2}+X_{L}{ }^{2}\right) \sigma_{x}{ }^{2}+R^{2} \sigma_{y}{ }^{2}\right]\left[\sigma_{x}{ }^{2}+\sigma_{y}{ }^{2}\right]}} .
\end{aligned}
$$

When we set $\sigma_{y}^{2}=0,(36)$ becomes

$$
\rho=\frac{2}{\pi} \sin ^{-1} \frac{1}{\sqrt{1+\left(\frac{X_{L}}{R_{L}}\right)^{2}}}
$$

and in particular when $X_{L}=0, \rho=1$ independent of $R_{L}$. This property that $\rho$ is independent of the load impedance and far-end talker power for a resistive load and no near-end talker does not hold when $u(t)$ and $v(t)$ are not limited, making $\rho$ more difficult to interpret.

The case where $\sigma_{y}{ }^{2}$ is appreciable can be detected from (36) by comparing $\rho$ to a threshold $\eta,-1<\eta<1$ :

$$
\rho<\eta
$$

since $\sigma_{y}{ }^{2}>0$ decreases $\rho$. Thus adaptation will be disabled whenever (38) is satisfied. In choosing $\eta$, we note that from (37) $\rho$ is also decreased even in the absence of a near-end talker when the load has a reactive component! Thus, for such a load, $\eta$ must be chosen less than (37) or adaptation will never be enabled. The smaller $\eta$, the larger the $\sigma_{y}{ }^{2}$ which will not be detected; that is, not satisfy (38). Hence, there is a tradeoff between the largest $\left(X_{\mathrm{L}} / R_{\mathrm{L}}\right)$ that is anticipated over all subscriber loops and the ability of the speech detector to detect small near-end talker powers.

Once $\eta$ is determined, the range of $\left(\sigma_{x}{ }^{2} / \sigma_{y}{ }^{2}\right)$ which will satisfy (38) can be determined from (36). As a numerical example we take the subscriber loop impedance data from Table I. For these data the largest $\left(X_{L} / R_{L}\right)$ is for the nonloaded cable at $3200 \mathrm{~Hz}$, for which from (37) $\rho=0.533$. Choosing a slightly smaller threshold $\eta=0.53$ we can calculate the largest $\left(\sigma_{x}{ }^{2} / \sigma_{y}{ }^{2}\right)$ for which (38) will be satisfied for each of the impedances of Table I. The result is summarized in Table III. For the case for which the threshold was picked, a nonloaded loop at $3200 \mathrm{~Hz}$, the speech detector is sensitive to a small near-end talker power $31 \mathrm{~dB}$ below the far-end talker power. The poorest discrimination is at the same frequency for a loaded loop, where a near-end talker power more than $5 \mathrm{~dB}$ below the far-end talker power will not be detected.

These results have been for the narrow-band signal case. However, wider bandwidth signals will lead to qualitatively similar results, since they will average the loop impedance characteristic over a band of frequencies. In addition, the finite return loss of the far-end hybrid as in Section III-F, which has not been considered here, will have an adverse effect on the operation of any speech detector.

\section{CONCLUSIONS}

The primary advantages of the adaptive balancing hybrid relative to an echo canceller for the local digital switch subscriber termination are simplicity of implementation, and the fact that a fair degree of balance is assured (without any special measures) regardless of the state of the adaptation. The adaptive balancing hybrid and echo canceller share the advantage 
TABLE III

SPEECH DETECTOR THRESHOLDS FOR $\eta=0.53$ AND IMPEDANCES OF TABLE I $(P=900 \Omega)$

\begin{tabular}{ccc}
\hline & & $10 \log \frac{\sigma_{\mathrm{x}}^{2}}{\sigma_{\mathrm{y}}^{2}}$ \\
\cline { 3 - 3 } & & $\frac{\text { Non-1oaded }}{2 \text { Loaded }}$ \\
0.2 & 9.17 & 6.25 \\
1.0 & 9.15 & 8.07 \\
3.2 & 30.96 & 5.01 \\
\hline
\end{tabular}

over other approaches of being able to maintain the best balance continuously in the face of changing conditions, such as different far-end talker spectrums, extension phones, and so forth. On the other hand, the other approaches do not require per-line circuitry.

The analysis of this paper has hopefully contributed to the understanding of the principles of operation and design tradeoffs of the adaptive balancing hybrid. It has uncovered no obstacles to its successful application to the local digital switch subscriber line interface.

Two versions of an adaptive balancing hybrid have been constructed and implementation details and experimental results are reported in a companion paper [11].

\section{APPENDIX A}

\section{SINGLE HYBRID ANALYSIS}

From Fig. 1(b), we can write immediately

$$
\begin{aligned}
& P(\theta)=\sigma_{y}{ }^{2}+\int_{-\infty}^{\infty} S_{x}(\omega)\left|H_{\mathrm{TH}}(\omega)\right|^{2} \frac{d \omega}{2 \pi} \\
& \sigma_{y}{ }^{2}=\int_{-\infty}^{\infty} S_{y}(\omega)\left|\frac{R}{R+Z_{L}}\right|^{2} \frac{d \omega}{2 \pi}
\end{aligned}
$$

where $\sigma_{y}{ }^{2}$ is the variance of the near-end talker (referenced to the four-wire transmit port) and it is assumed that $x(t)$ and $y(t)$ are uncorrelated. Equation (A.1) motivates us to define

$$
\begin{aligned}
& (G, H)=\int_{-\infty}^{\infty} S_{x}(\omega) H(\omega) G^{*}(\omega) \frac{d \omega}{2 \pi} \\
& \|H\|^{2}=(H, H) .
\end{aligned}
$$

Readers familiar with Hilbert space notions will recognize (A.2) as an $L_{2}$ inner product (with weighting function $S_{x}(\omega)$ ); other readers can simply view (A.2),(A.3) as a convenient shorthand notation. In view of (2), (3) and (A.1)

$$
P(\theta)=\sigma_{y}^{2}+\left\|H_{L}-H\right\|^{2} .
$$

In view of the interpretation of $\left\|H_{L}-H\right\|^{2}$ as the length of a

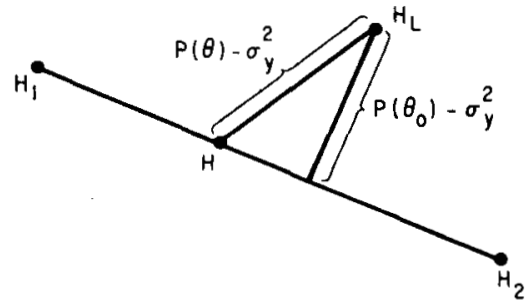

Fig. 7. Geometric interpretation of $P(\theta)$.

vector $\left(H_{L}-H\right),(\mathrm{A} .4)$ assumes the geometric significance of Fig. 7. As $\theta$ goes from 0 to $1, H$ moves from $H_{2}$ to $H_{1}$, and $P(\theta)-\sigma_{y}{ }^{2}$ is the distance from $H_{L}$ to $H$. The minimum $P(\theta)$, $P\left(\theta_{0}\right)$, is evidently reached when the vector $H_{L}-H$ is orthogonal to the vector $\left(H_{1}-H_{2}\right)$ :

$$
\left(H_{L}-H, H_{1}-H_{2}\right)=0, \theta=\theta_{0}
$$

but using (2), (A.5) can be used to solve for $\theta_{0}$ :

$$
\theta_{0}=\frac{\left(H_{L}-H_{2}, H_{1}-H_{2}\right)}{\left\|H_{1}-H_{2}\right\|^{2}} .
$$

The constant $K$ can be determined by applying the Pythagorean rule to the triangle in Fig. 3

$$
\begin{aligned}
&\left\|H_{L}-\theta H_{1}-(1-\theta) H_{2}\right\|^{2} \\
&=\left\|\theta H_{1}+(1-\theta) H_{2}-\theta_{0} H_{1}-\left(1-\theta_{0}\right) H_{2}\right\|^{2} \\
&+\left\|H_{L}-\theta_{0} H_{1}-\left(1-\theta_{0}\right) H_{2}\right\|^{2}
\end{aligned}
$$

which yields (5) with

$$
K=\left\|H_{1}-H_{2}\right\|^{2}
$$

which is recognized as the variance of $w(t)$. Finally, $P\left(\theta_{0}\right)$ can be determined by substituting (A.6) into (A.4), with the result

$$
P\left(\theta_{0}\right)=\sigma_{y}{ }^{2}+\left\|H_{L}-H_{2}\right\|^{2}-\frac{\left(H_{L}-H_{2}, H_{1}-H_{2}\right)^{2}}{\left\|H_{1}-H_{2}\right\|^{2}} .
$$

It is hardly surprising to note that the Schwarz inequality, when applied to (A.8), yields

$$
P\left(\theta_{0}\right) \geqslant \sigma_{y}^{2}
$$

with equality if and only if $H_{L}$ is of the form of (2) for some $\theta$.

\section{APPENDIX B}

VARIANCE OF $Q(\theta)$

Case $I-(x(t)$ Sinusoidal)

When $x(t)$ is given by (14), $w(t)$ is also sinusoidal with amplitude $A\left|H_{1}\left(\omega_{0}\right)-H_{2}\left(\omega_{0}\right)\right|$ and $z(t)$ has two terms, one 
sinusoidal which will result in dc $Q(\theta)$ plus a $2 \omega_{0}$ frequency term which can be ignored in $q(t)$ (since it will be well outside the bandwidth of the closed loop transfer function for practical values of $\mu$ ), and the other $y(t)$. The latter contributes to $S_{q}(\omega)$, and can easily be shown to be

$$
\begin{aligned}
S_{q}(\omega)= & \frac{1}{4} A^{2}\left|H_{1}\left(\omega_{0}\right)-H_{2}\left(\omega_{0}\right)\right|^{2} . \\
& \cdot\left\{S_{y}\left(\omega+\omega_{0}\right)+S_{y}\left(\omega-\omega_{0}\right)\right\} .
\end{aligned}
$$

\section{Case II- $(y(t)$ Sinusoidal)}

When $y(t)$ assumes the form of (14), w(t) has power spectrum

$$
S_{w}(\omega)=\left|H_{1}(\omega)-H_{2}(\omega)\right|^{2} S_{x}(\omega)
$$

and, similarly to (B.1)

$$
S_{q}(\omega)=\frac{1}{4} A^{2}\left\{S_{w}\left(\omega+\omega_{0}\right)+S_{w}\left(\omega-\omega_{0}\right)\right\}
$$

\section{Case III- $(x(t)$ and $y(t)$ Gaussian)}

When $x(t)$ and $y(t)$ are independent stationary Gaussian random processes, $q(t)$ has autocorrelation [10]

$$
R_{q}(\tau)=R_{z}(\tau) R_{w}(\tau)+R_{z w}(-\tau) R_{z w}(\tau)
$$

and thus

$$
\begin{aligned}
S_{q}(0) & =\int_{-\infty}^{\infty} R_{q}(\tau) d \tau \\
& =\int_{-\infty}^{\infty}\left(S_{z}(\omega) S_{w}(\omega)+S_{z w}{ }^{2}(\omega)\right) \frac{d \omega}{2 \pi} \\
S_{z}(\omega) & =S_{y}(\omega)+\left|H_{\mathrm{TH}}(\omega)\right|^{2} S_{x}(\omega), \\
S_{w}(\omega) & =S_{x}(\omega)\left|H_{1}(\omega)-H_{2}(\omega)\right|^{2}, \\
S_{z w}(\omega) & =S_{x}(\omega) H_{\mathrm{TH}}{ }^{*}(\omega)\left(H_{1}(\omega)-H_{2}(\omega)\right) .
\end{aligned}
$$

Substituting (B.6)-(B.8) into (B.5), we obtain (17)-(19).

\section{APPENDIX C}

\section{EFFECT OF LIMITING ON ADAPTATION}

The impact of limiting either $w(t)$ or $z(t)$ or both on the adaptation will now be evaluated by assuming that $w(t)$ and $z(t)$ are stationary and Gaussian. It follows directly from Price's theorem [6] that

$$
\begin{aligned}
& E(w(t) \operatorname{sgn} z(t))=\sqrt{\frac{2}{\pi}} Q(\theta) / \sigma_{z}, \\
& E(z(t) \operatorname{sgn} w(t))=\sqrt{\frac{2}{\pi}} Q(\theta) / \sigma_{w}, \\
& E(\operatorname{sgn} w(t) \operatorname{sgn} z(t))=\frac{2}{\pi} \sin ^{-1}\left(\frac{Q(\theta)}{\sigma_{w} \sigma_{z}}\right) .
\end{aligned}
$$

Thus, limiting both signals results in a nonlinear relationship between $Q(\theta)$ and its estimate, but the linear relationship is retained if only one signal is limited. Substituting from (4), (5), (6), and (9) we obtain

$$
\begin{aligned}
& E(z(t) \operatorname{sgn} w(t))=\sqrt{\frac{2}{\pi}} \sigma_{w}\left(\theta_{0}-\theta\right), \\
& E(w(t) \operatorname{sgn} z(t))=\sqrt{\frac{2}{\pi}} \frac{\sigma_{w}{ }^{2}\left(\theta_{0}-\theta\right)}{\sqrt{P\left(\theta_{0}\right)+\sigma_{w}{ }^{2}\left(\theta_{0}-\theta\right)^{2}}},
\end{aligned}
$$

$E(\operatorname{sgn} w(t) \operatorname{sgn} z(t))$

$$
=\frac{2}{\pi} \sin ^{-1}\left(\frac{\sigma_{w}\left(\theta_{0}-\theta\right)}{\sqrt{P\left(\theta_{0}\right)+\sigma_{w}^{2}\left(\theta_{0}-\theta\right)^{2}}}\right) .
$$

When $P\left(\theta_{0}\right)$ is small, (C.5), (C.6) simplify to

$$
\begin{aligned}
& E(w(t) \operatorname{sgn} z(t)) \cong \sqrt{\frac{2}{\pi}} \sigma_{w} \operatorname{sgn}\left(\theta_{0}-\theta\right) \\
& E(\operatorname{sgn} w(t) \operatorname{sgn} z(t)) \cong \operatorname{sgn}\left(\theta_{0}-\theta\right)
\end{aligned}
$$

and in both cases all information about the magnitude of the error $\left(\theta_{0}-\theta\right)$ is lost. When $P\left(\theta_{0}\right)$ is large relative to $\sigma_{w}{ }^{2}$ $\left(\theta_{0}-\theta\right)^{2}$, such as when there is a large near-end talker or $\theta$ is near its optimum value (C.5), (C.6) simplify to

$$
\begin{aligned}
& E(w(t) \operatorname{sgn} z(t)) \cong \sqrt{\frac{2}{\pi P\left(\theta_{0}\right)}} \sigma_{w}{ }^{2}\left(\theta_{0}-\theta\right) \\
& E(\operatorname{sgn} w(t) \operatorname{sgn} z(t)) \cong \frac{2}{\pi} \sin ^{-1}\left(\frac{\sigma_{w}\left(\theta_{0}-\theta\right)}{\sqrt{P\left(\theta_{0}\right)}}\right) .
\end{aligned}
$$

In both cases the cross correlation tends to zero as $P\left(\theta_{0}\right)$ increases even if $\theta$ is not optimum. Thus, limiting $z(t)$ results in a desirable automatic desensitization of the adaptation when there is a large near-end talker, but loss of information about the magnitude of $\left(\theta_{0}-\theta\right)$ in the absence of a near-end talker.

The effect of limiting $w(t)$ or $z(t)$ or both on the rate of adaptation can now be established. When $w(t)$ alone is limited, $K$ in (10) is proportional to $\sigma_{w}$ rather than $\sigma_{w}{ }^{2}$ :

$$
K=\sqrt{\frac{2}{\pi}} \sigma_{w}
$$

reducing somewhat the dependence of the speed of adaptation on far-end talker power. However, the variance of $\theta$ in (13) is now more sensitive to $\sigma_{w}$, having terms proportional to $\sigma_{w}$ and $\sigma_{w}{ }^{2}$.

When $z(t)$ is limited and $\left(\dot{\theta}_{0}-\theta\right)$ is large, $(10)$ is no longer valid and $\theta(t)$ changes linearly in $t$ with slope $C \mu$, where

$$
C=\sqrt{\frac{2}{\pi}} \sigma_{w}
$$


when $w(t)$ is not limited, and

$$
C=1
$$

when $w(t)$ is also limited. As $\theta(t)$ approaches $\theta_{0},(10)$ becomes valid again with

$$
K=\sqrt{\frac{2}{\pi P\left(\theta_{0}\right)}} \sigma_{w}^{2}
$$

when $w(t)$ is not limited, and

$$
K=\frac{2}{\pi} \frac{\sigma_{w}}{\sqrt{P\left(\theta_{0}\right)}}
$$

when $w(t)$ is limited. The variance of (13) remains valid when $\theta$ is near $\theta_{0}$, with the $K$ given by (C.14), (C.16). In view of (A.4), when there is no near-end talker $P\left(\theta_{0}\right)$ is proportional to the power in $x(t)$, so that (C.14) is effectively proportional to $\sigma_{w}$ and (C.15) is effectively independent of $\sigma_{w}{ }^{2}$. Thus, the effect of limiting $z(t)$ alone on the variance of (13) is about the same as limiting $w(t)$ alone, whereas limiting both $w(t)$ and $z(t)$ results in a larger sensitivity to the power in $x(t)$. When there is a near-end talker and $\sigma_{y}{ }^{2}$ in (A.4) is large, $K$ in both (C.14) and (C.15) approaches zero, and the variance of $\theta$ in (13) becomes very large.

\section{ACKNOWLEDGMENT}

The author appreciates the useful discussions and collaboration he had with Prof.D.A. Hodges, A. de la Plaza, and B. Dotter during the course of this work.

\section{REFERENCES}

[1] L. S. DiBiaso, " Transmission considerations for local switched digital network," Telephony, p. 40, Oct. 24, 1977.

[2] J. L. Neigh, "Transmission planning for an evolving local switched digital network," IEEE Trans. Commun. . vol. COM-27, p. 1019. July 1979
[3] R. L. Bunker, F. J. Scida, and R. P. McCabe, "Line matching networks to support zero loss operation in digital class 5 offices." in Conf. Rec., Int. Conf. Subscriber Loops and Systems, Atlanta, GA, Mar. 1978.

[4] _-. "Zero loss considerations in a digital class 5 office," IEEE Trans. Commun., vol. COM-27, p. 1013, July 1979.

15] D. L. Duttweiler, "A twelve-channel digital echo canceler," IEEE Trans. Commun., vol. COM-26, p. 647, May 1978.

[6] R. Price, "A useful theorem for nonlinear devices having Gaussian inputs," IRE Trans. Inform. Theory, p. 69, June 1958

[7] P. A. Gresh, "Physical and transmission characteristics of customer loop plant," Bell Syst. Tech. J., p. 3337, Dec. 1969.

[8] A. de la Plaza, "Auto-balancing hybrid for telephone lines," M.S thesis, Univ. California at Berkeley, Berkeley, CA, Dec. 1978.

[9] W. C. Ahern, F. P. Duffy, and J. A. Maher, "Speech signal power in the switched message network," Bell Syst. Tech. J., p. 2695, Sept. 1978.

[10] W. B. Davenport and W. L. Root, Random Signals and Noise. New York: McGraw-Hill, 1958.

[11] B. Dotter, A. de la Plaza, D. A. Hodges, and D. G. Messerschmitt, "Implementation of an adaptive balancing hybrid," this issue. pp. $1408-1416$.

[12] M. M. Sondi and D. A. Berkley, "Silencing echos on the telephone network," Proc. IEEE, to be published.

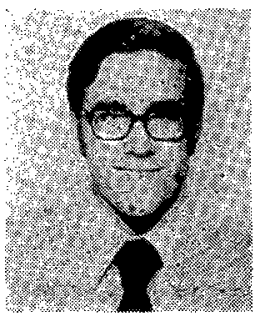

David G. Messerschmitt (S'65-M'68-SM'78) received the $B . S$. degree from the University of Colorado, Boulder, in 1967, and the M.S. and Ph.D. degrees from the University of Michigan. Ann Arbor, in 1968 and 1971, respectively.

Since 1977 he has been an Associate Professor of Electrical Engineering and Computer Sciences at the University of California at Berkeley. From 1968 to 1974 he was a member of the Technical Staff, and from 1974 to 1977 he was a Supervisor at Bell Laboratories, Holmdel. NJ, where he did systems engineering, development, and research on digital transmission lines and terminals, digital speech interpolation, and digital signal processing. particularly as it relates to both low and high bit rate encoding of speech. Since 1977 he has served as a consultant primarily to TRW VIDAR, Hughes Aircraft, and Acumenics, and also to TRW Systems, TRW Electronics, California Microwave, L. M. Ericsson, Siemens, and Intel.

Dr. Messerschmitt is a member of Eta Kappa Nu. Tau Beta Pi, and Sigma Xi. He is currently serving as Editor for Transmission Systems of the IEEE TRANSACTIONS ON COMMUNICATIONS. 